\title{
PDE4D is a therapeutic target in melanoma
}

洲 Inserm

Institut national

\author{
Nicolas Dumaz ${ }^{1,2}$, Julie Delyon ${ }^{1,2,3}$, Selma Becherirat ${ }^{1,2}$, Jocelyne André ${ }^{1,2}$, Céleste Lebbé ${ }^{1,2,3}$ \\ ${ }^{1}$ INSERM U976, Skin Research Institute, Paris, France \\ ${ }^{2}$ UMRS976, Paris Diderot University, Sorbonne Paris Cité, Paris, France \\ ${ }^{3}$ Dermatology Department, Saint Louis Hospital, Paris, France
}

\section{INTRODUCTION}

The figure on the right indicates the three major signaling pathways involved in melanoma. Proteins indicated in red are coded by genes which are mutated, amplified or deleted in melanoma. The cAMP pathway (MC1R/PKA/CREB/MITF) is both important for normal melanocyte biology and implicated in melanoma development. We have previously shown that transformation of melanocytes by oncogenic BRAF, NRAS or KIT inhibits CAMP signaling. This inhibition involves the phosphodiesterase-4 (PDE4) enzymes that physiologically degrade CAMP.

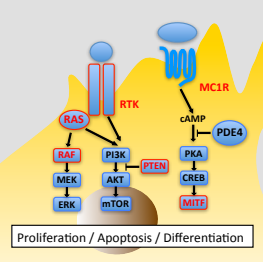

\section{RESULTS}

PDE4D expression is increased in patients with advanced melanoma

We analyzed the expression of PDE4D in 43 human melanoma tumors (33 primary melanomas and 10 lymph node metastases) by quantitative RT-PCR and immunohistochemistry. PDE4D mRNA and protein were expressed more abundantly in advanced stages of melanoma.
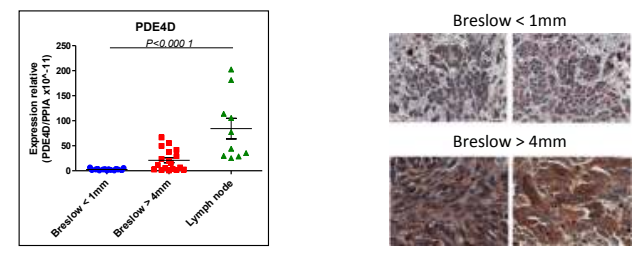

\section{PDE4D promotes melanoma invasion by interacting with FAK}

Under conditions of suboptimal adenylyl cyclase activity (Forskolin 1uM), inhibition of PDE4 (Rolipram 10 uM; F1R10) can suppress the invasion of 4 different BRAF-mutated melanoma cell lines by 50 to $80 \%$. More precisely, we further showed that PDE4D promoted melanoma invasion by interacting with focal adhesion kinase (FAK) through the scaffolding protein RACK1 (Delyon et al. Oncogene 2017).
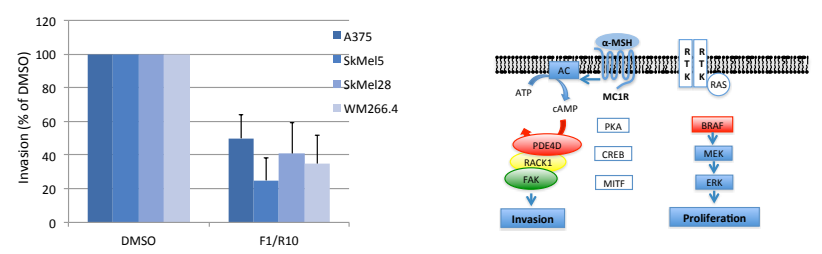

\section{CONCLUSION}

PDE4D may serve as a new therapeutic target for the treatment of aggressive melanoma and may prevent the emergence of melanoma clones with enhanced metastatic capabilities. PDE4 inhibitors are already available in the clinics and could be used as a complementary therapeutic approach.
3 Inhibition of PDE4 reduces growth of melanoma cells in 2D and 3D

Inhibition of PDE4 (Forskolin 1uM + Rolipram $10 \mathrm{uM}$; F1R10) reduced clone formation in the SkMel28 BRAF-mutated melanoma cell lines more efficiently than a low dose of the BRAF inhibitor vemurafenib (3uM; V3). Inhibition of PDE4 also reduced the growth of two BRAF-mutated melanoma cell lines grown in suspension as melanospheres.
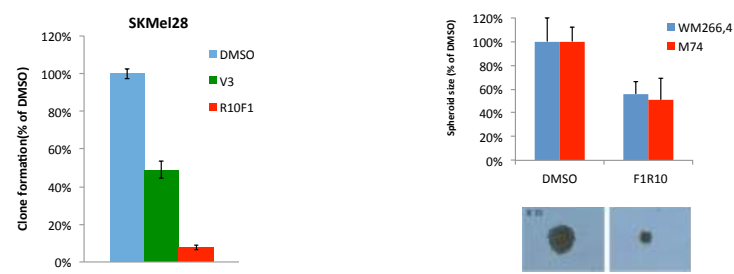

PDE4 inhibitors inhibit proliferation of melanoma cell lines resistant to BRAF inhibitors

We generated 2 independent melanoma cell lines derived from SKMel28 with acquired resistance to vemurafenib (SKMel28-Res and SKMel28-RAS). Resistance resulted in maintenance of ERK phosphorylation and proliferation in the presence of vemurafenib at a concentration sufficient to inhibit parental cells (V3 and V10). Inhibition of PDE4 with three different inhibitors (Rolipram, Apremilast or Roflumoilast) resulted in strong reduction of clone formation in resistant lines even in the absence of forskolin.
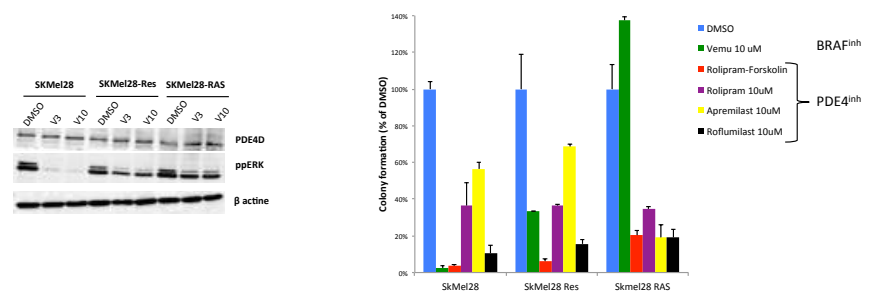

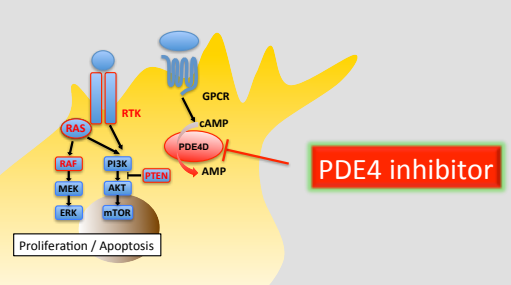

